\title{
Nanocomposite coatings obtained by electrophoretic co-deposition of poly(etheretherketone)/graphene oxide suspensions
}

\author{
Eduin I. González-Castillo ${ }^{1}$ (D), Tommaso Costantini ${ }^{2}$ (D), Milo S. P. Shaffer ${ }^{2,3}$ (D), and \\ Aldo R. Boccaccini ${ }^{1, *}$ (1) \\ ${ }^{1}$ Institute of Biomaterials, University of Erlangen-Nuremberg, Cauerstrasse 6, 91058 Erlangen, Germany \\ ${ }^{2}$ Department of Materials, Imperial College London, South Kensington Campus, London SW7 2AZ, UK \\ ${ }^{3}$ Department of Chemistry, Imperial College London, South Kensington Campus, London SW7 2AZ, UK
}

Received: 7 January 2020

Accepted: 31 March 2020

Published online:

20 April 2020

(C) The Author(s) 2020

\begin{abstract}
Nanocomposite coatings were successfully prepared by electrophoretic deposition of poly(etheretherketone) (PEEK)/graphene oxide (GO) suspensions. The GO flakes developed a large-scale co-continuous morphology with the basal plane mainly aligned with the coating surface. However, the PEEK particles were also found to be wrapped by GO nanosheets when deposited on the stainless steel substrate. Both phenomena, the co-continuous morphology and the wrapping effect, were dependent on the initial GO content in the suspension and influenced the final morphological characteristics of the thermally treated coatings. The PEEK matrix developed a dendritic morphology during its cooling from the molten state because of transcrystallinity that was induced by the incorporation of GO. The preparation of suspensions involved tip ultrasonication (TS) to deagglomerate, disperse, and mill the PEEK particles. A detailed study of the microstructure revealed that TS tended not only to reduce PEEK particle size, but also to promote an elongated shape, favourable for the nanocomposite coatings.
\end{abstract}

\section{Introduction}

Poly(oxy-1,4-phenyleneoxy-1,4-phenylenecarbonyl1,4-phenylene) or poly(etheretherketone) (PEEK) is a high-performance and semicrystalline thermoplastic polymer in the polyaryletherketone family, which exhibits outstanding properties such as chemical, tribological, hard radiation and creep resistance as well as high strength and temperature stability, biocompatibility, low flammability without the necessity of flame-retardant additives, and low smoke emission [1-5]. As a result of its properties, PEEK finds a broad range of applications, ranging from medicine to aerospace and nuclear industry [5]. Particularly in the case of coating applications, PEEK-based coatings

Address correspondence to E-mail: aldo.boccaccini@ww.uni-erlangen.de 
systems possess good adhesion to metallic substrates, and at the same time, they enhance the substrate resistance against wear and corrosion [6]. Electrophoretic deposition (EPD) is an effective processing method for coating deposition on a variety of substrates, offering a number of advantages such as excellent mass and thickness control, relative lowcost, and large range of materials suitable to be used, including biomaterials, composites, and nanocomposites $[7,8]$. EPD is a two-step colloidal process that involve electrophoresis, the migration of charged particles in a liquid suspension towards an electrode by effect of an electric field, and deposition of the particles on the electrode, forming a relatively dense coating [9].

Polymer matrix composites that incorporate fillers in the nano- or micrometric scale with the aim of tailoring desired properties (including mechanical and functional properties as well as processability) are a leading area of current materials research and development [10-13]. Carbonaceous, ceramic and metallic fillers have been used to tailor the properties of PEEK-based coatings obtained by EPD to specific requirements. Nanocomposite performance depends on different factors such as dispersion, matrix-filler interactions, and, in case of flake and fibre reinforcements, alignment [10]. In this respect, EPD is useful to develop specific microstructures from multicomponent suspensions [14]; for instance, in the study of PEEK/MoS $\mathrm{M}_{2}$ coatings by electrophoretic codeposition, $\mathrm{MoS}_{2}$ was found to align preferentially, parallel to the coating surface [15]. Similar preferential alignment of graphene oxide (GO) nanosheets has been reported in the EPD process [16-21]. Furthermore, the incorporation of GO has two main advantages: firstly, the adhesion of GO coatings on metallic substrates after thermal treatment has been attributed to the interaction between the oxygenated functional groups of GO and the metallic surface, and thereby, GO coatings have been used as the interface between PEEK and the metallic substrate to enhance the mechanical properties [21]; secondly, $\pi$-conjugated structures in graphitic materials can form strong $\pi-\pi$ stacking interactions with the benzene ring such that found in PEEK $[22,23]$. Nonetheless, direct co-deposition of PEEK/GO by EPD, which should be an attractive route to nanocomposite coatings, has not been explored so far. GO possesses a two-dimensional (2D) flat monolayer formed from crystalline domains of $\mathrm{sp}^{2}$-hybridised carbon atoms in a hexagonal lattice, as well as amorphous/defective domains associated with a large number of $\mathrm{sp}^{3} \mathrm{C}-\mathrm{O}$ bonds, incorporated in epoxide, carbonyl, carboxyl, and hydroxyl groups [24-26]. GO has attracted enormous interest in recent years for its outstanding properties, including Young's modulus, strength, high specific surface area, molecular barrier properties, remarkable optical properties, and excellent thermal and electrical conductivity [26]. Because of its structural characteristics and outstanding properties, GO has been implemented as filler in the development of numerous polymer matrix nanocomposite systems for several applications [27-31].

Tip ultrasonication (TS) is frequently implemented to facilitate particle deagglomeration and dispersion of nanofillers, as a crucial step in the production of high-quality nanocomposites. However, it has also been used for purification, particle size reduction (in sizes ranging from nano- to micrometres), and development of specific particle morphology $[32,33]$ and sonochemical synthesis [34-36]. Despite its benefits, TS has been rarely used in preparing PEEK suspensions for EPD [37-39] in comparison with bath ultrasonication [6, 40-53].

In this work, nanocomposite coatings are obtained by EPD from PEEK/GO suspensions and subsequent thermal treatment. PEEK/GO suspensions are prepared in two stages: (1) TS of PEEK particles is explored as a means to optimise their shape, size, and aspect ratio, by varying the applied oscillation amplitude; (2) GO is incorporated to obtain the PEEK/GO suspensions. The micromorphology and microstructure of the resulting PEEK/GO coatings are analysed before and after the thermal treatment. The results indicate that GO has an important role in the micromorphology of the coatings, as GO nanosheets develop a large-scale co-continuous morphology characterised by the basal plane of the nanosheets mainly aligned with the coating surface, and the wrapping of PEEK particles by GO nanosheets. Moreover, incorporation of GO promotes the transcrystallinity of PEEK after melting.

\section{Experimental}

\section{Materials}

VICTREX $^{\mathrm{TM}}$ PEEK VICOTE ${ }^{\mathrm{TM}} 704$ (particle size $10-50 \mu \mathrm{m}$, density $1.32 \mathrm{~g} \cdot \mathrm{cm}^{-3}$, glass transition 
temperature, $T_{\mathrm{g}}, 143{ }^{\circ} \mathrm{C}$, and melting temperature, $T_{\mathrm{m}}, 343{ }^{\circ} \mathrm{C}$ ) recommended as coating of ferrous and non-ferrous metals was acquired from Victrex PCL, UK. Single-layer graphene oxide (flake size $0.5-2.0 \mu \mathrm{m}$, thickness $0.6-1.2 \mathrm{~nm}$, and single-layer ratio $>80 \%$ ) dispersed in deionised water, concentration of $10 \mathrm{mg} \cdot \mathrm{mL}^{-1}$, was purchased from ACS Materials, USA. The suspension medium components, ethanol denatured with about $1 \%$ methyl ethyl ketone for analysis, and isopropanol, 99.9\% purity, were supplied by EMSURE ${ }^{\circledR}$ and Sigma-Aldrich, respectively. Stainless steel $316 \mathrm{~L}$ foil (Thyssenkrupp, Germany) was used as work and counter electrodes during EPD, dimensions $15 \mathrm{~mm} \times 2 \mathrm{~mm}$. Physical properties and chemical composition of stainless steel $316 \mathrm{~L}$ are listed in Table 1 [54]. VICTREX ${ }^{\mathrm{TM}}$ PEEK VICOTE $^{\mathrm{TM}} 704$, graphene oxide, and stainless steel $316 \mathrm{~L}$ are hereafter referred as PEEK, GO, and stainless steel, respectively.

\section{Methods}

The process to co-deposit PEEK/GO composite coatings introduced in this work is schematised in Fig. 1. A two-stage processing method was developed in order to prepare and optimise the multicomponent suspensions for EPD (Fig. 1a, b). Firstly, PEEK suspensions were obtained by adding the polymer powder, $3 \mathrm{wt} \%$, into $40 \mathrm{~mL}$ of a co-solvent formed by ethanol and isopropanol, 95 and $5 \mathrm{vol} \%$, respectively. PEEK particles were deagglomerated, dispersed, and milled using a $20 \mathrm{kHz}$ Digital Sonifier ${ }^{\circledR}$ S250D disruptor (BRANSON Ultrasonic Corporation, USA) equipped with a tapered and 4.7-mmdiameter titanium microtip (Fig. 1a). TS parameters considered were pulse mode, $0.5 \mathrm{~s}$ "on" followed by $2.1 \mathrm{~s}$ "off", and effective TS time of $6 \mathrm{~min}$ at constant amplitude. Amplitudes values of 30 and $60 \%$ were selected to determine the effect of TS on the PEEK particle size. The microtip was submerged into the PEEK suspension until the microtip edge was $1 \mathrm{~cm}$ above the bottom of the $50-\mathrm{mL}$ glass beaker. During TS, PEEK suspensions were cooled down to an ice bath, and their temperature was monitored in real time, at a sample rate of $4 \mathrm{~S} / \mathrm{s}$, with a type $\mathrm{K}$ thermocouple (RS Components $\mathrm{GmbH}$, Germany) connected to a single-channel thermocouple module USB-TC01 (National Instruments, UK), and logged every second. PEEK suspension temperature remained below $20^{\circ} \mathrm{C}$ in all cases. The acoustic energy delivered to the ethanol/isopropanol medium was estimated following the calorimetric method [55]; more details can be found in the Supporting Information. Delivered acoustic power values of $1.5 \mathrm{~W}$ and $3.8 \mathrm{~W}$ for $30 \%$ and $60 \%$ amplitude, respectively, were obtained by applying the following equation:

$P=\mathrm{d} / \mathrm{d} t(T) \cdot M \cdot C_{\mathrm{p}}$

where $P$ is the delivered acoustic power (W), $T$ corresponds to the temperature $(\mathrm{K}), t$ represents the time (s), and $M(\mathrm{~g})$ and $C_{p}\left(\mathrm{~J} \cdot \mathrm{g}^{-1} \mathrm{~K}^{-1}\right)$ are the mass of the liquid and specific heat of liquid, respectively. By plotting the temperature against time (Fig. S1-Supplementary Information), the value of $\mathrm{d} / \mathrm{dt}(T)$ was estimated considering the linear regression slope. The $C_{p}$ value was proportional to the ethanol/isopropanol mixture ratio, ethanol $95 \mathrm{vol} \%$ and isopropanol $5 \mathrm{vol} \%, C_{p}$ being $2.36 \mathrm{~J} \cdot \mathrm{g}^{-1} \cdot \mathrm{K}^{-1}$ for ethanol and $2.57 \mathrm{~J} \cdot \mathrm{g}^{-1} \cdot \mathrm{K}^{-1}$ for isopropanol $[56,57]$.

In the second stage, sonicated PEEK and as-delivered GO suspensions were blended at three different compositions (Fig. 1b, c). The concentration of the starting PEEK suspension was kept constant, and a

Table 1 Stainless steel $316 \mathrm{~L}$ chemical composition and properties

\begin{tabular}{|c|c|c|c|c|c|c|c|}
\hline \multicolumn{8}{|l|}{ Property } \\
\hline $\begin{array}{l}\text { Density } \\
\left(\mathrm{kg} \cdot \mathrm{m}^{-3}\right)\end{array}$ & $\begin{array}{l}\text { Thermal } \\
\text { conductivity } \\
\left(\mathrm{W} \cdot \mathrm{m}^{-1} \cdot \mathrm{K}^{-1}\right)\end{array}$ & $\begin{array}{l}\text { Elastic } \\
\text { modulus } \\
(\mathrm{GPa})\end{array}$ & $\begin{array}{l}\text { Specific thermal } \\
\text { capacity } \\
\left(\mathrm{J} \cdot \mathrm{kg}^{-1} \cdot \mathrm{K}^{-1}\right)\end{array}$ & $\begin{array}{l}\text { Electrical } \\
\text { resistivity } \\
\left(\Omega \cdot \mathrm{mm}^{2} \cdot \mathrm{m}^{-1}\right)\end{array}$ & $\begin{array}{l}\text { Linear thermal } \\
\text { expansion coefficient } \\
\left(10^{-6} \cdot \mathrm{K}^{-1}\right)\end{array}$ & $\begin{array}{l}\text { Yield } \\
\text { strength } \\
(\mathrm{MPa})\end{array}$ & $\begin{array}{l}\text { Tensile } \\
\text { strength } \\
(\mathrm{MPa})\end{array}$ \\
\hline 8.0 & 15 & 200 & 500 & 0.75 & 16.0 & 220 & $520-670$ \\
\hline
\end{tabular}

All properties are reported for a temperature of $20{ }^{\circ} \mathrm{C}$ 


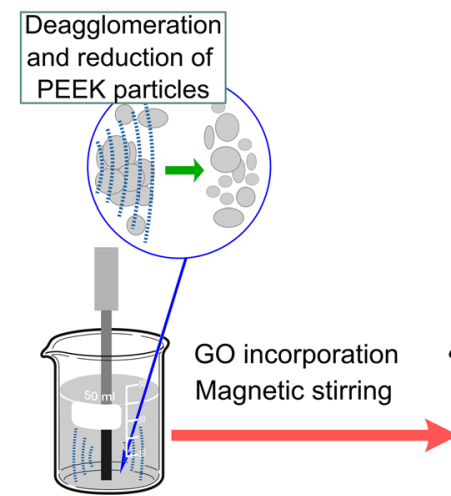

Tip sonication of PEEK suspension

(a) (b) (c)

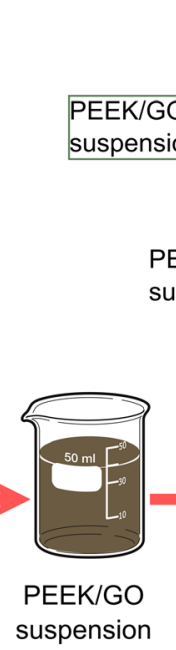

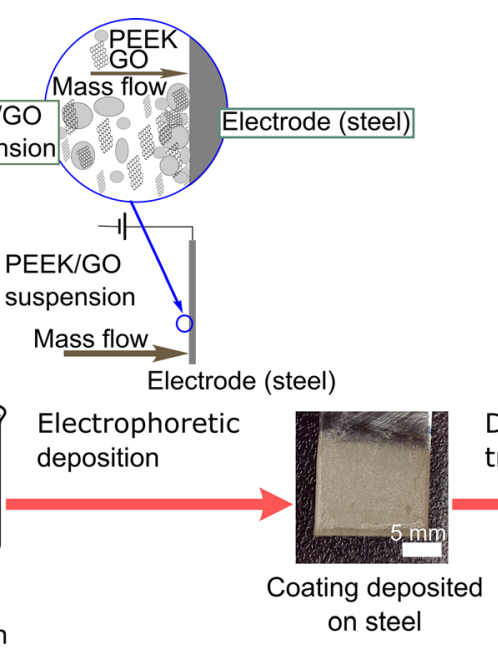

(d) (e)

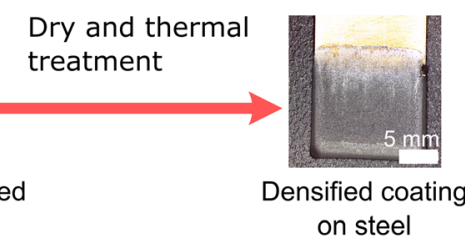

(f)

(g)

Figure 1 Scheme of the process followed for preparation of co-deposited PEEK/GO coatings.

different volume of GO suspension was added for the preparation of each blend. The GO suspension was carefully transferred with a pipette from its original container to the previously ultrasonicated PEEK suspension and magnetically stirred on a stirring hotplate (VMS-C7 Advanced VWR ${ }^{\mathrm{TM}}$, USA) for $24 \mathrm{~h}$ at $250 \mathrm{rpm}$ and ambient temperature. Blends at compositions of $0.5,1.0$ and $3.0 \mathrm{wt} \% \mathrm{GO}$ relative to the PEEK mass were prepared adding, respectively, $0.5,1.0$, and $3.0 \mathrm{~mL}$ of GO suspension (1 wt\%) to $40 \mathrm{~mL}$ of PEEK suspensions (3 wt $\%$ ). Table 2 summarises the characteristics of the suspensions prepared and their nomenclature used hereafter to identify them.

EPD experiments were carried out at constant voltage and ambient temperature (Fig. 1d). The microstructural homogeneity and thickness of the coating heavily influenced by voltage. Thereby, in order to observe the effect of voltage on co-deposited PEEK/GO coatings, 10 and $30 \mathrm{~V}$ were considered for EPD and adjusted using a DC power supply EL752M multimode (Thurlby Thandar Instruments, UK). Stainless steel electrode plates of dimensions (30 $\mathrm{mm} \times 15 \mathrm{~mm} \times 2 \mathrm{~mm})$ were mirror-polished with a P600 CarbiMetTM grinding paper (Bühler Group, Switzerland) and cleaned for $30 \mathrm{~min}$ in an ethanolic medium by means of a SONOREX super RK 100 (BANDELIN electronic GmbH \& Co. KG, Germany) before EPD to improve the adhesion of the coating to the stainless steel substrate. Two stainless steel electrodes were submerged into the PEEK or PEEK/GO suspension at the centre of a borosilicate beaker in a parallel plate arrangement $(10 \mathrm{~mm}$ distance between plates) to achieve a uniform electric field between the plates. Deposition times from 1 to 5 min were considered because some PEEK particles formed a thin layer at the bottom of the beaker after 8 min due to sedimentation; nevertheless, low turbidity was achieved after $40 \mathrm{~min}$, and the suspensions were suitable for EPD. After deposition, the coated electrode plates were carefully extracted from the suspension to prevent damage to the deposited

Table 2 Nomenclature used to designate the PEEK/GO suspensions, the EPD experiments, and the thermally treated coatings

\begin{tabular}{|c|c|c|c|c|c|c|}
\hline \multicolumn{5}{|l|}{ Suspension } & \multirow{2}{*}{$\begin{array}{l}\text { EPD coating } \\
\text { nomenclature }\end{array}$} & \multirow{2}{*}{$\begin{array}{l}\text { Thermally treated } \\
\text { coating } \\
\text { nomenclature }\end{array}$} \\
\hline Nomenclature & $\begin{array}{l}\text { PEEK to } \\
\text { suspension } \\
\text { medium (wt } \%)\end{array}$ & $\begin{array}{l}\text { GO suspension } \\
\text { to suspension } \\
\text { medium (vol\%) }\end{array}$ & $\begin{array}{l}\text { GO to PEEK } \\
(\mathrm{wt} \%)\end{array}$ & $\begin{array}{l}\text { Solid } \\
\text { content }(\%)\end{array}$ & & \\
\hline PEEK/0.5GO & 3 & 1.25 & 0.5 & 3.01 & PEEK/0.5GO_EPD & PEEK/0.5GO_TT \\
\hline PEEK/1.0GO & 3 & 2.50 & 1.0 & 3.03 & PEEK/1.0GO_EPD & PEEK/1.0GO_TT \\
\hline PEEK/3.0GO & 3 & 7.50 & 3.0 & 3.09 & PEEK/3.0GO_EPD & PEEK/3.0GO_TT \\
\hline
\end{tabular}


layer, Fig. 1e. The EPD coatings were labelled with reference to the suspension used for their preparation, see Table 2.

Prior to thermal treatment, Fig. 1f, samples were dried at $150{ }^{\circ} \mathrm{C}$ for $3 \mathrm{~h}$, according to the recommendations given by the manufacturer, in a muffle furnace P330 (Nabertherm GmbH, Germany). Thermal treatment was carried out in air applying the following thermal cycle: heating from room temperature to $380{ }^{\circ} \mathrm{C}$ at a rate of $10^{\circ} \mathrm{C} \cdot \mathrm{min}^{-1}$, followed by an isothermal step at $380{ }^{\circ} \mathrm{C}$ for $5 \mathrm{~min}$; finally, cooling from $380{ }^{\circ} \mathrm{C}$ to room temperature at a rate of $2{ }^{\circ} \mathrm{C} \cdot \mathrm{min}^{-1}$. Instruments described previously to monitor the temperature during sonication were also used to monitor the temperature in real time during the drying and thermal treatment stages, and the temperature values were logged every minute in both cases. A representative sample after drying and thermal treatment is presented in Fig. 1g. Table 2 lists the nomenclature followed to designate the thermally treated coatings; in all cases, the nomenclature is associated with the suspension from which the coating was obtained.

\section{Characterisation}

\section{Scanning electron microscopy}

Scanning electron microscopy (SEM) images were taken on a field-emission microscope ZEISS Gemini SEM 450 (ZEISS, Germany) equipped with an in-lens secondary electron detector. Graphene oxide is highly electron transparent as a result of its 2D structure and low atomic number, and it can be transparent when high accelerating voltages are set because the incident electrons pass more easily thorough the GO nanosheets [58]. Hence, an accelerating voltage of $1 \mathrm{kV}$ and probe current of $10 \mathrm{nA}$ were selected to favour the observation of GO nanosheet details and to ensure comparability between the materials prepared. SEM images were taken for raw PEEK particles and PEEK/GO composite coatings; the latter ones were examined before and after the thermal treatment. Before SEM analysis, samples were dried at $60{ }^{\circ} \mathrm{C}$ for $24 \mathrm{~h}$ in a drying oven with natural convection (DL 115 DRY-line ${ }^{\circledR}$, VWR International BVBA, Belgium) and subsequently coated with $\mathrm{Au}$ into a sputter coater (Q150T S Plus, Quorum, UK) to prevent sample charging.
With the aim of determining the TS effect on the PEEK particle characteristics, volumes of $50 \mu \mathrm{L}$ of PEEK suspensions tip-ultrasonicated at different amplitudes were gently deposited on aluminium conductive tape with a micro-pipette to determine the effect of TS on the characteristics of PEEK particles. A detailed analysis of the SEM micrographs of the deposited PEEK particles was performed by means of ImageJ software (version 1.52a) and Wolfram Mathematica ${ }^{\circledR}$ (version 8.0.0.0). For every single particle, the contour was traced, and the minor and major particle axes were determined using ImageJ software. Representative results were ensured by considering over 400 particles for SEM micrograph. In addition, bivariate probability density distributions (BPDDs) were obtained. BPDD comprised a total of 1600 subregions, 40 horizontal subintervals, and 40 vertical ones. Kernel density estimation (KDE) was calculated considering Silverman's rule and Gaussian kernel [59] by implementing Wolfram Mathematica ${ }^{\circledR}$.

\section{Fourier-transform infrared spectroscopy}

Infrared spectra were recorded in the wavenumber interval from 4000 to $600 \mathrm{~cm}^{-1}, 40$ scans, $4 \mathrm{~cm}^{-1}$ resolution and $4.3 \mathrm{~cm}$ aperture, by using an IRTracer100 (Shimadzu Corp., Japan) Fourier transform infrared (FTIR) spectrometer equipped with a highenergy ceramic light source and a deuterated L-alanine triglycine sulphate (DLATGS) detector. FTIR spectrometer was coupled to a single reflection attenuated total reflectance (ATR) system (Quest, Specac Ltd., UK), $45^{\circ}$ nominal angle of incidence, equipped with a diamond prism. Samples were placed on the diamond prism and pressed to its surface by mean of compression rod. Spectrum software (version 10.02.00.0041) was used in the analysis of the ATR-FTIR spectra.

\section{X-ray diffraction}

X-ray diffraction (XRD) characterisation of PEEK and PEEK/GO coatings was carried out at room temperature by using a Rigaku Miniflex ${ }^{\mathrm{TM}} 600$ (Rigaku, Japan) X-ray diffractometer provided with a $\mathrm{Cu}-\mathrm{K} \alpha$ radiation $(\lambda=1.5406 \AA)$ X-ray source and scintillation counter ( $K \beta$ filter) detector. The instrument was operated at $40 \mathrm{kV}$ and $15 \mathrm{~mA}$. XRD patterns were recorded from the specimen surface in the angular 


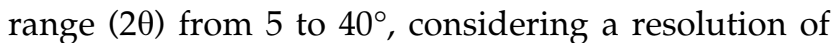
$0.01^{\circ}$ and speed scanning of $0.5^{\circ} \cdot \mathrm{min}^{-1}$.

\section{Results and discussion}

\section{Effect of tip ultrasonication on the PEEK particles}

Acoustic cavitation produced during TS promotes highly intense local conditions characterised by short-lifetime hot spots whose temperature rises to $5000{ }^{\circ} \mathrm{C}$, reaching pressure values of around $1000 \mathrm{~atm}$, and cooling rates of $10^{10} \mathrm{~K} \cdot \mathrm{s}^{-1}$ [60]. Even though propagation of ultrasonic waves in liquids is generally considered as an adiabatic process because of the rapid pressure variation [61], the highly intense local conditions can cause ceramic, metallic, carbonaceous, and polymeric particles to experience, according to their nature, changes of their molecular weight, chemical and crystalline structure, and morphology [32, 33, 61-67]. Hence, the first step was to determine the effects of TS on PEEK particles.

In principle, FTIR spectroscopy can be used to monitor variations in the molecular weight, and chemical and crystalline structure. FTIR spectra of neat PEEK particles is presented in Fig. 2a. The thermal degradation mechanism of PEEK involves random scission of ketone and ether linkages [68-70], leading to the disappearance of characteristic PEEK bands in mid-infrared (MIR) at $1652 \mathrm{~cm}^{-1} v_{(\mathrm{C}=\mathrm{O})}$, $1225 \mathrm{~cm}^{-1} v_{(\varphi-\mathrm{O}-\varphi)}, 1010 \mathrm{~cm}^{-1} v_{(\mathrm{C}-\mathrm{O}-\mathrm{C})}$ or $v_{(\mathrm{C}-\mathrm{O})}$, and

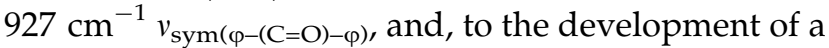
band at $1709 \mathrm{~cm}^{-1}$ related to an aldehyde group, which is well defined even at weight loss values as low as 5\% [71]. In contrast to thermal degradation, the ultrasonic degradation of polymers in solution is coupled to a non-stochastic mechano-chemical shear; long polymer chains are, therefore, degraded much more rapidly than small ones, resulting in average molecular weight $\left(M_{\mathrm{w}}\right)$ reduction at earlier stages [61]. In the present experiments, PEEK particles were insoluble in the ethanol/isopropanol medium, and the differences in the spectra for the neat PEEK and processed polymer after TS at constant amplitude values of 30 and $60 \%$ were modest (Fig. 2a). Moreover, there was no evidence of new bands associated with aldehyde or other groups as possible result of a thermal degradation mechanism; however, there was a slight intensity reduction and shift of PEEK characteristic bands after TS (see inset in Fig. 2a), with increasing amplitude. This behaviour can be attributed to $M_{\mathrm{w}}$ reduction of the PEEK particles, and, in last instance, to a small contribution from ultrasonic degradation of PEEK. Similar results have been found in ultrasonication of chitosan nanoparticles [66].

Crystallinity plays an important role in semicrystalline polymers, not only because it affects their final properties, but also for the EPD process itself. Amorphous polymer chains extending out of the particle surface make a major contribution to the suspension stability [48]. The behaviour of insoluble polymer particles in suspensions is similar to that of solid particles such as metals and ceramics with adsorbed polymers on their surfaces [72-74]. TS is known to modify material crystallinity in some cases [33], but the effects on PEEK are not yet established. FTIR provides an alternative to estimate the degree of crystallinity; in case of PEEK, it is through the MIR bands at $1305 \mathrm{~cm}^{-1} \quad v_{(\mathrm{C}=\mathrm{O}),} \quad 1280 \mathrm{~cm}^{-1} \quad v_{(\mathrm{C}=\mathrm{O})}$, $966 \mathrm{~cm}^{-1} \omega_{(\mathrm{CH})}$, and $952 \mathrm{~cm}^{-1} \omega_{(\mathrm{CH})}$ [75-79]. By using these bands, Chalmers et al. [75] and Cebe et al. [77] established calibration curves to quantify the degree of crystallinity of PEEK from FTIR measurements. In this work, the calibration curve for the $\mathrm{C}-\mathrm{H}$ wagging mode reported by Cebe et al. [77] is applied, as it uses the band area rather than intensity ratio and was cross-checked against a range of techniques to determine the degree of crystallinity. According to the FTIR measurements and the selected calibration curve, no significant variations in the degree of crystallinity of PEEK were detected under the TS experimental conditions (Table 3). The degree of crystallinity was also estimated from XRD measurements: Fig. $2 b$ shows XRD patterns of the pristine PEEK powder before and after tip sonication considering amplitudes of 30 and $60 \%$. PEEK has an orthorhombic unit cell where the polymer backbones are aligned with the c-axis, giving rise to four main peaks in the XRD diffractogram at $2 \theta$ values of approximately $19^{\circ}, 21^{\circ}, 23^{\circ}$, and $29^{\circ}$, assigned to the (110), (111), (200), and (211) crystalline planes, respectively [80-83]. The degree of crystallinity for pristine and tip sonicated PEEK powders was estimated from XRD measurements according to the procedure reported in the literature [81, 83]; the values are summarised in Table 3. A comparison between the degree of crystallinity measured by XRD and deduced from FTIR calibration curve [77] shows 
Figure 2 FTIR spectra and XRD patterns of neat PEEK particles, and PEEK particles after ultrasonication at amplitude of 30 and $60 \%$.
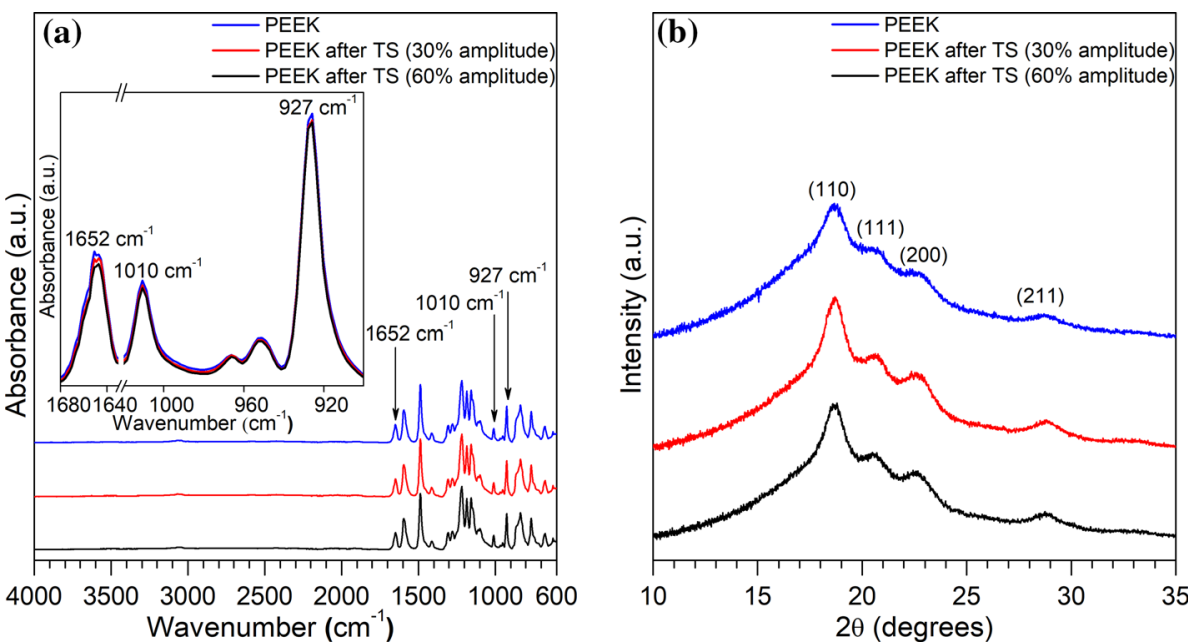

Table 3 Estimation of the degree of crystallinity of PEEK according to a calibration curve reported by Cebe et al. [77], and XRD measurements

\begin{tabular}{llll}
\hline Amplitude (\%) & FTIR & & XRD \\
\cline { 2 - 4 } & $\begin{array}{l}\text { Band area ratio } \\
966 \mathrm{~cm}^{-1} / 952 \mathrm{~cm}^{-1}\end{array}$ & $\begin{array}{l}\text { Degree of crystallinity (\%) } \\
\text { from calibration curve [77] }\end{array}$ & $\begin{array}{l}\text { Degree of } \\
\text { crystallinity (\%) }\end{array}$ \\
\hline 0 & 0.3340 & 18 & 20 \\
30 & 0.3648 & 21 & 23 \\
60 & 0.3401 & 19 & 19 \\
\hline
\end{tabular}

The considered MIR bands correspond to $\omega(\mathrm{CH})$ modes

a good correlation between the two methods and suggests that the effect of TS on the crystallinity of PEEK is minor and can be neglected.

In addition to the particle's structure, its overall morphology is a key factor in the processing and properties of composites and nanocomposites. During TS, polymer particles may change morphology, depending on both process parameters (power, amplitude, suspension concentration, suspension volume, and microtip diameter/length immersed) and the characteristics of the particles (initial size, and their chemical and physical characteristics). TS is commonly associated with reduction of particle size; however, under some conditions, TS increases the polymer particle size; for example, an increase in particle size has been observed in TS of poly(methyl methacrylate) particles, where the authors attributed the behaviour to the fusion of the beads in dumbbelllike structures [65]. Light-scattering (LS) techniques have been used to estimate PEEK particle size after TS; nevertheless, agglomeration and precipitation of the particles cause fluctuations in the measurements
$[37,39]$. Furthermore, the effects of TS on PEEK particle shape have not been reported; thus, a detailed SEM study was performed here. A large number of SEM images (up to 10 images, more than 400 particles analysed in each image) was obtained (typical example in Fig. 3a) and analysed quantitatively to obtain a BPDD which offers valuable information about how the variables interact or are correlated with each other (Fig. 3b). The initial PEEK particles are defined by major and minor particle size values $\leq 40 \mu \mathrm{m}$. Tick marks situated close to the BPDD axes show the locations of the individual observations. The diagonal line indicates the location of equiaxed particles, with an aspect ratio of 1 . Rounded particles (e.g. particle numbered 1 in Fig. 3a) appear near the line in Fig. 3b, and elongated particles (e.g. particle number 2 in Fig. 3a) lie further away. There are relatively fewer larger particles (major axis over $\sim 20 \mu \mathrm{m}$ ), but those that are present tend to be more elongated (Fig. $3 b$ ).

Despite the fact that BPDD provides valuable information, noise in the measurement process and 

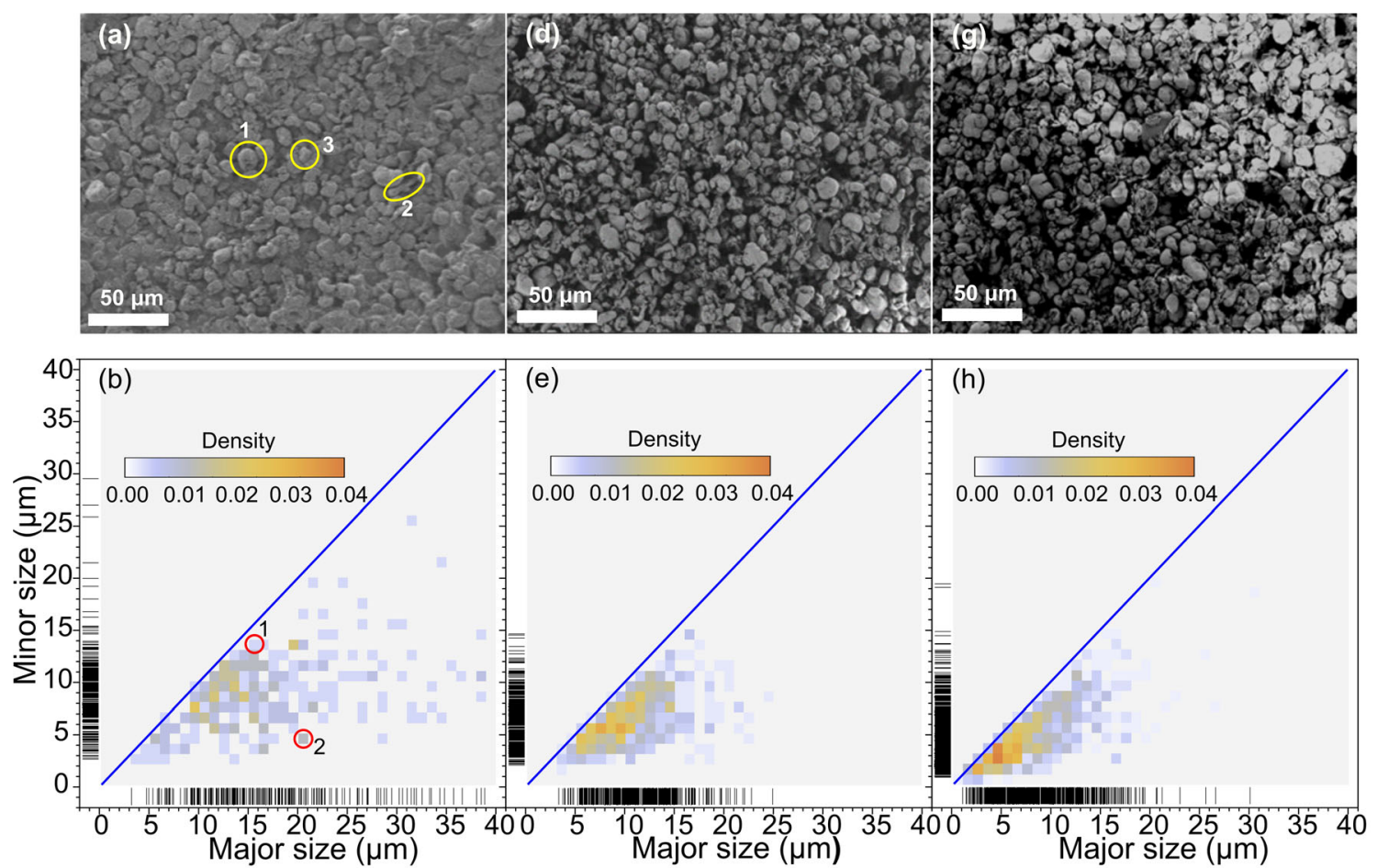
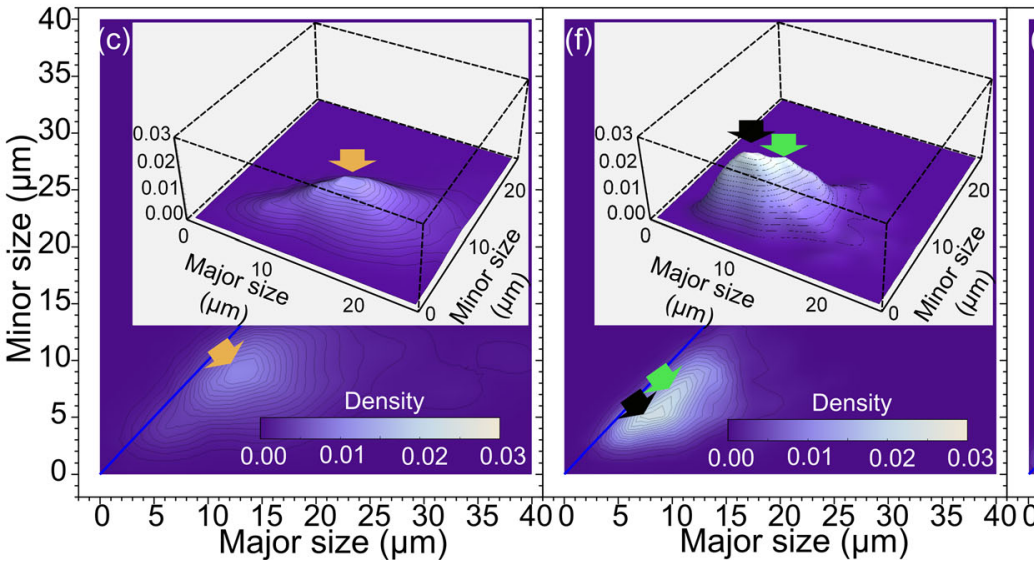

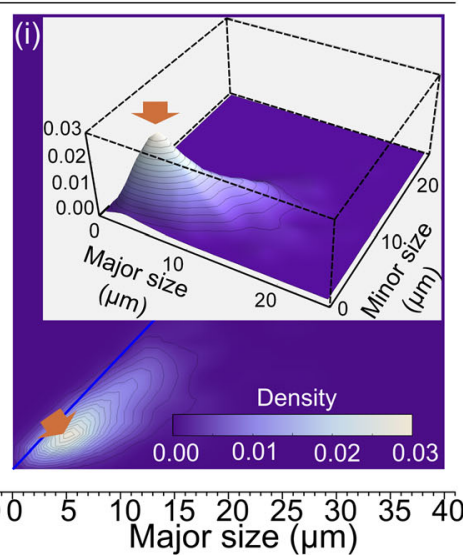

Figure 3 SEM micrographs of neat PEEK particles (a), and PEEK particles after TS at constant amplitude of $30 \%$ (d) and $60 \%$ (g). DPDDs and KEDs are shown in $\mathbf{b}$, $\mathbf{e}$, and $\mathbf{h}$, and $\mathbf{c}, \mathbf{f}$, and $\mathbf{i}$,

subinterval size can influence the result; for example, the probability that an observation lies in a subinterval depends on the subinterval size selected. By contrast, the use of KDE reduces these effects, allowing a fundamental data smoothing where inferences about the population are made [84]. KDE values derived from the individual observations are shown in Fig. 3c. The representation of the experimental observations in terms of KDE shows a distinct broad peak, indicated by the arrow, whose coordinates are related to the estimated particle sizes with the highest probability. This peak is centred at $13 \mu \mathrm{m}$ respectively. Circles marked (1), (2), (3) in a refer to different PEEK particle types, as described in the text.

(major size) and $9 \mu \mathrm{m}$ (minor size) values, implying an average aspect ratio around 1.4. An archetypal PEEK particle with the characteristics described above is indicated in Fig. 3a as number 3.

After TS, both BPDD and KDE show a significant reduction in modal particle size, as well as an increase in particles smaller than $20 \mu \mathrm{m}$ (Fig. 3). KDE (Fig. 3i) highlights that particles below $5 \mu \mathrm{m}$ are the dominant ones after the higher amplitude $(60 \%)$ treatment. At 30\% amplitude (Fig. 3f), the distribution is bimodal, and the larger peak shifts to lower size values in respect to the original particle 
distribution. The shifts of distribution were observed to be correlated with the amplitude (Fig. 3f, i); nevertheless, the particle size was reduced by only $30 \%$ though the amplitude was doubled. This behaviour is consistent with that observed in polymers and other materials, where the rate decreases with particle size as consequence of energy lost in particle motion, compression, flexing, and other mechanisms rather than fragmentation [50]. Although the LS measurements in the case of PEEK particles are time dependent because of particle agglomeration and precipitation, the particle sizes determined by SEM are consistent with the range of values determined by means of LS of a few to tens of microns [37, 39]. Interestingly, TS generates more elongated PEEK particles, with the aspect ratio increasing from 1.4 for the original powder to 1.75 to 2.5 when the amplitude is increased from 30 to $60 \%$, respectively. Table 4 lists additional statistical parameters for neat PEEK and tip-ultrasonicated PEEK particles.

After TS, the PEEK particle dimensions are closer to the GO flake size, $0.5-2.0 \mu \mathrm{m}$, and have a more elongated shape, which may be an advantage in their interaction with the 2D nanosheets. Although there were only minimal changes in PEEK molecular weight and crystallinity, extended TS can be undesirable. High amplitudes increase the unavoidable erosion of the microtip, introducing impurities and contaminating the suspension. Therefore, a 30\% constant amplitude was selected for subsequent processing of the PEEK powder.

\section{PEEK/GO coatings}

The suspensions of PEEK particles and GO were mixed and then deposited by EPD onto stainless steel substrates, to form high-quality, uniform coatings. SEM micrographs show that the GO nanosheets are homogeneously distributed across the coating (Fig. 4) and also suggest two characteristic modes of interaction between the two phases after EPD and subsequent drying process: nanosheets deposited on the top or trapped in between the PEEK particles (Fig. 4a, b); and nanosheets wrapping the particles (Fig. 4c). Most importantly, SEM micrographs indicate that GO nanosheets form a co-continuous structure (Fig. $4 \mathrm{~d}-$ f). The large GO nanosheet structures have dimensions exceeding the flake size indicated by the manufacturer, $0.5-2.0 \mu \mathrm{m}$, suggesting an assembly process during the EPD. GO continuous morphologies [85] and GO alignment have been reported [86]. Nevertheless, in the present conditions, though it is plausible that the position of the GO nanosheets could differ from the horizontal position, it is remarkable that the GO nanosheets basal plane is predominantly aligned with the nanocomposite coating surface, forming structures whose dimensions are larger than several thousands of $\mu \mathrm{m}$. SEM at increasing magnification shows the assembly process in more detail (indicated by the arrows in Fig. $4 \mathrm{~d}-\mathrm{f}$ ).

Alignment of GO nanosheets, as result of EPD, has been discussed in the literature [17-20]. One explanation is that the basal plane will align to the direction of the applied electric field during EPD due to the anisotropic polarizability of the GO nanosheets; another possibility is that the GO aligns parallel to the substrate either due to rotation during deposition or due to capillary effects during drying. Large and aligned discontinuous GO structures were formed with $0.5 \mathrm{wt} \%$ GO loading in suspension (Fig. 5a, d), but as the GO content increased to $1.0 \mathrm{wt} \%$, the cocontinuous morphology appeared (Fig. 5b, e), becoming more prominent at $3.0 \mathrm{wt} \%$ (Fig. 5c, f).

Regarding PEEK particle size and aspect ratio after $E P D$, it is not straightforward to quantify these parameters from SEM micrographs. Even by increasing the accelerating voltage from 1 to $10 \mathrm{kV}$ (Fig. 6) with the aim of varying the transparency of

Table 4 Statistical parameters obtained for PEEK powder as function of the TS amplitude

\begin{tabular}{|c|c|c|c|c|c|c|c|}
\hline \multirow[t]{3}{*}{ Amplitude } & \multicolumn{7}{|c|}{ Statistical parameter } \\
\hline & \multicolumn{2}{|l|}{ Mean } & \multicolumn{2}{|l|}{ Median } & \multirow[t]{2}{*}{ Covariance } & \multirow[t]{2}{*}{ Skewness } & \multirow[t]{2}{*}{ Kurtosis } \\
\hline & Major size $(\mu \mathrm{m})$ & Minor size $(\mu \mathrm{m})$ & Major size $(\mu \mathrm{m})$ & Minor size $(\mu \mathrm{m})$ & & & \\
\hline 0 & 17.7 & 9.2 & 15.1 & 8.7 & 38.8 & 52.6 & 89.0 \\
\hline 30 & 10.8 & 6.4 & 10.1 & 6.2 & 5.0 & 2.4 & 10.0 \\
\hline 60 & 8.5 & 4.9 & 7.3 & 4.3 & 9.2 & 4.0 & 13.5 \\
\hline
\end{tabular}



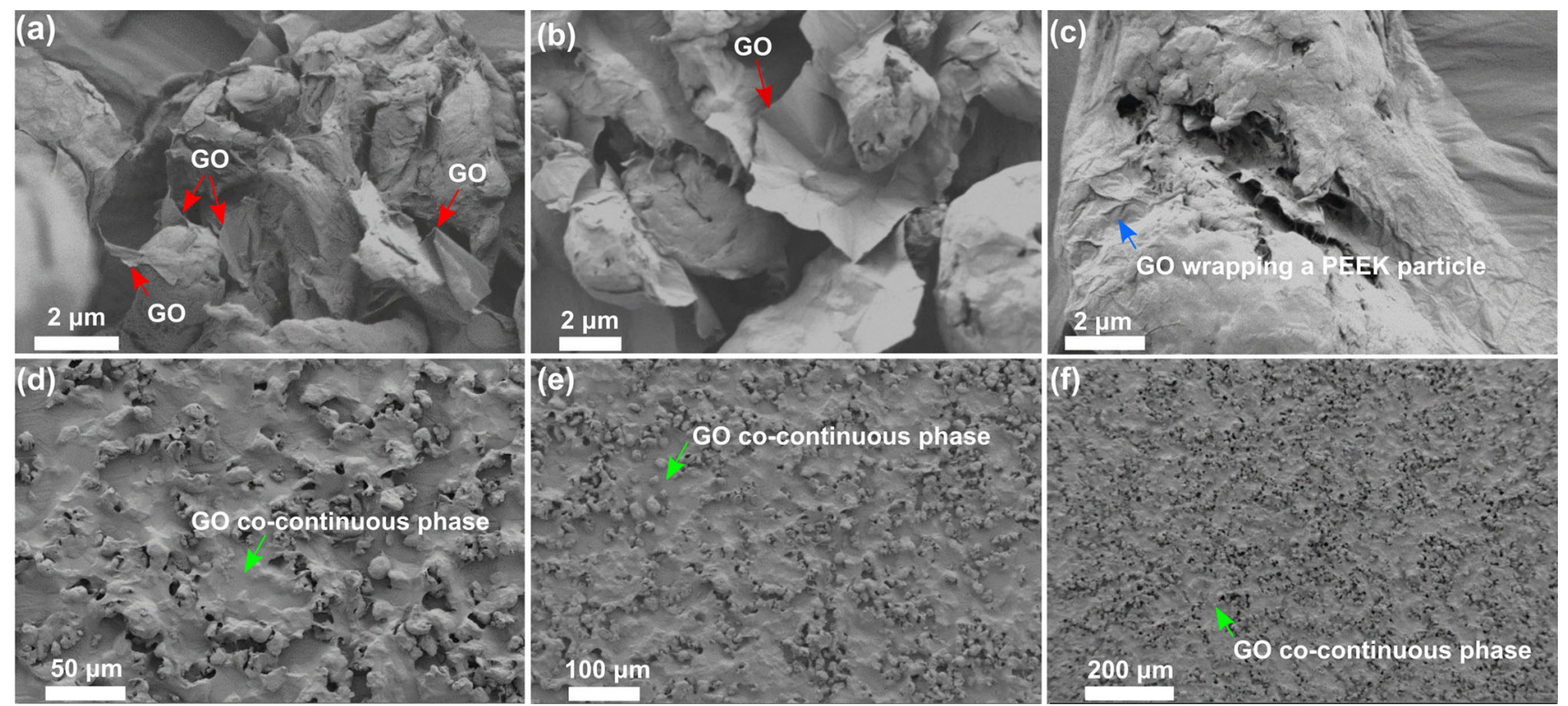

Figure 4 SEM micrographs of a nanocomposite coating PEEK/3.0GO_EPD. GO nanosheets form a co-continuous morphology, with their basal plane mainly aligned with the coating surface. EPD parameters: $10 \mathrm{~V}$ and deposition time of $1 \mathrm{~min}$.

the GO nanosheets to the electron beam [58, 87], allowing PEEK particles to be visualised underneath, GO flakes are seen to cover the particles, specially small particles. Nonetheless, the majority of the observable deposited PEEK particles have a size below $10 \mu \mathrm{m}$, exhibiting elongated shape of low aspect ratio (Fig. $5 \mathrm{~d}-\mathrm{f}$ ), although there was a considerable population of particles (after TS) with major size ranging between 10 and $20 \mu \mathrm{m}$ after TS (Fig. 3d- f). This effect is likely associated with the sedimentation of large PEEK particles during EPD. Composition, particle size and shape, and size distribution are key factors dictating the success of EPD [88, 89], confirming that TS is an effective strategy to prepare suitable suspensions containing PEEK powders and GO for EPD.

Macroscopically, PEEK/GO nanocomposite coatings deposited at $30 \mathrm{~V}$ were, in general, more
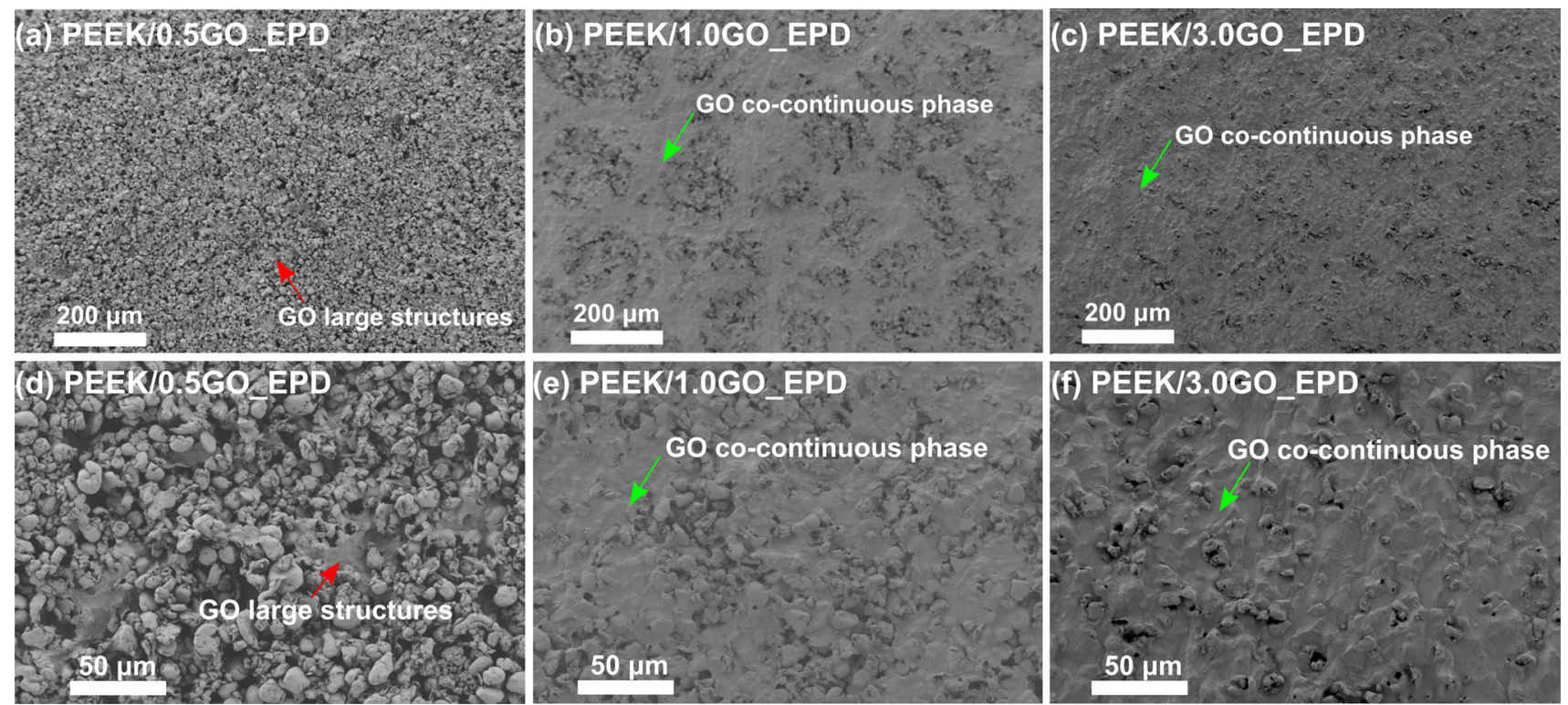

Figure 5 SEM micrographs of coatings PEEK/GO_EPD obtained from PEEK/GO suspensions with different GO concentrations. The respective $\mathrm{GO} \mathrm{wt} \%$ ratio is indicated in the sample codification on each micrograph. EPD parameters: $30 \mathrm{~V}$ and 3-min deposition time.

\section{Springer}



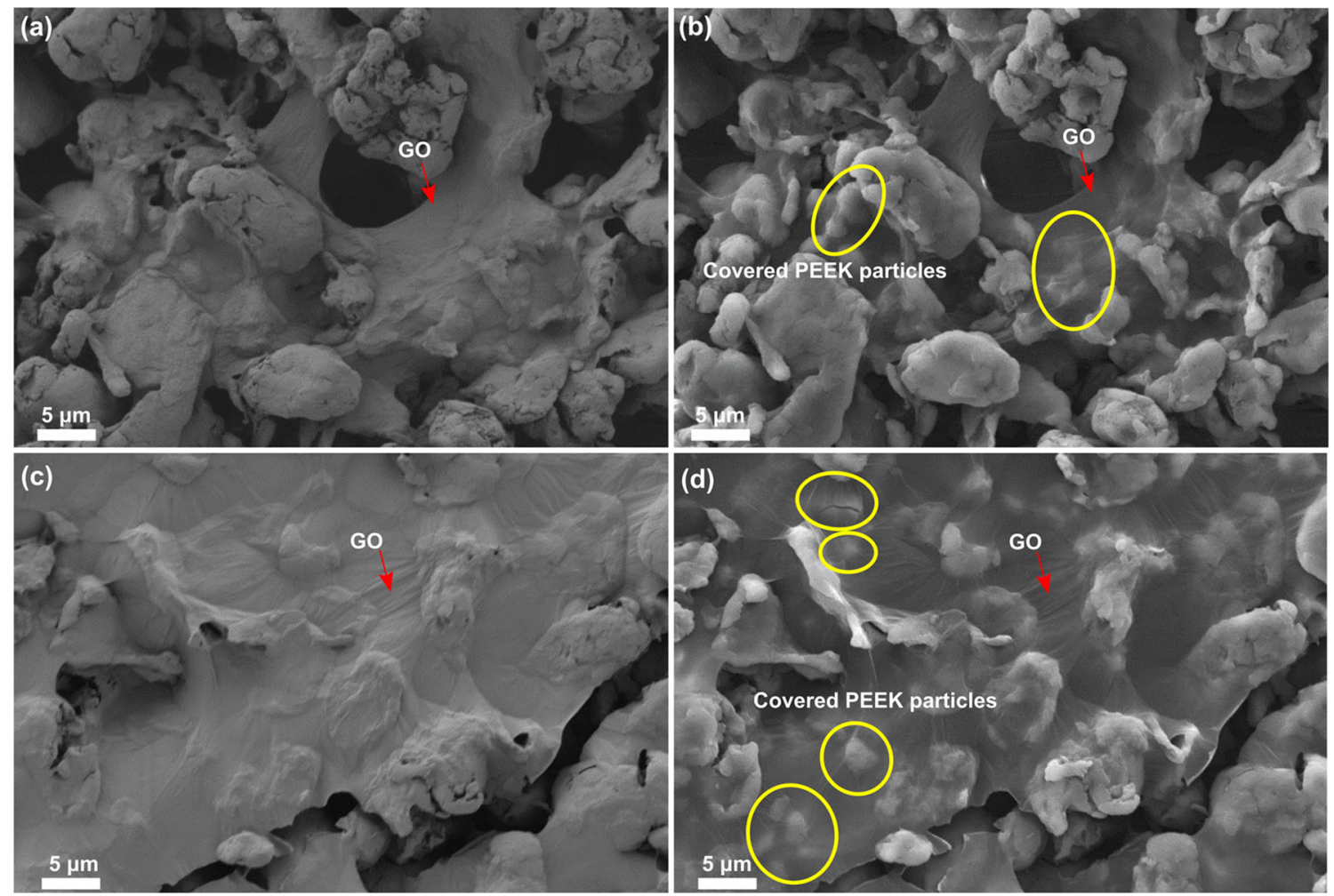

Figure 6 SEM images of PEEK/3.0GO_EPD in two different regions at different accelerating voltages: $\mathbf{a}$ and $\mathbf{c} 1.0 \mathrm{kV}$, and $\mathbf{b}$ and d $10.0 \mathrm{kV}$. EPD parameters: $10 \mathrm{~V}$ and deposition time of $1 \mathrm{~min}$.

uniform and thicker than those deposited at $10 \mathrm{~V}$. Nevertheless, there was a significant edge effect when deposition time was extended over $4 \mathrm{~min}$ (Fig. 7), especially when the GO concentration increased in the suspension, leading to crack formation along the substrate edges. Therefore, samples prepared by EPD at $30 \mathrm{~V}$ and 3 min were selected for further analysis.

Electrophoretic deposition of PEEK requires a postsintering process in order to densify the coating, as well as to enhance the adhesion to the stainless steel substrate [40-42, 44, 45, 49]. Digital images of the dried and thermally treated PEEK/GO coatings (Fig. 8) show coherent coatings. PEEK/0.5GO_TT and PEEK/1.0GO_TT were uniform in the centre of the sample, Fig. 8a, b, but a darker area was visible close to the substrate edges in both coatings. In contrast, PEEK/3.0GO_TT was relatively uniform. These macroscopic features may be related to the wettability and viscosity of the melted PEEK, being both phenomena, wettability and viscosity, dependent on the heat-treatment temperature [90]. Moreover, GO can substantially increase the viscosity in polymers above the rheological percolation threshold [91], and, in PEEK/GO composites, the viscosity could be increased one order of magnitude with respect to neat PEEK even at GO concentrations lower than 5\% [92]. This suggests that the nanosheets impede the free spreading of the melted PEEK over the surface, specially, at the edges (Fig. 8), where it is likely that more GO is deposited due to field enhanced deposition (Fig. 7).

The uniform central region of the consolidated PEEK/GO_TT coatings (Fig. 8) was studied by SEM, XRD, and FTIR to explore the effects of GO. Increasing GO content creates a rougher, more irregular surface (Fig. 9a-c). These irregularities can be attributed to the GO nanosheet structure. PEEK particles wrapped by GO nanosheets after EPD (Fig. 4c) may not be able to integrate into the continuous film during melting. Comparison of the SEM micrographs of Fig. 5d-f with those in Fig. 9a-c shows that the size and distribution of the particles before thermal treatment correspond with the observed irregularities. Furthermore, the texture of the co-continuous GO phase may be also manifested in the coating surface (compare Fig. 5). In addition to the micron-scale texture, a finer dendritic 


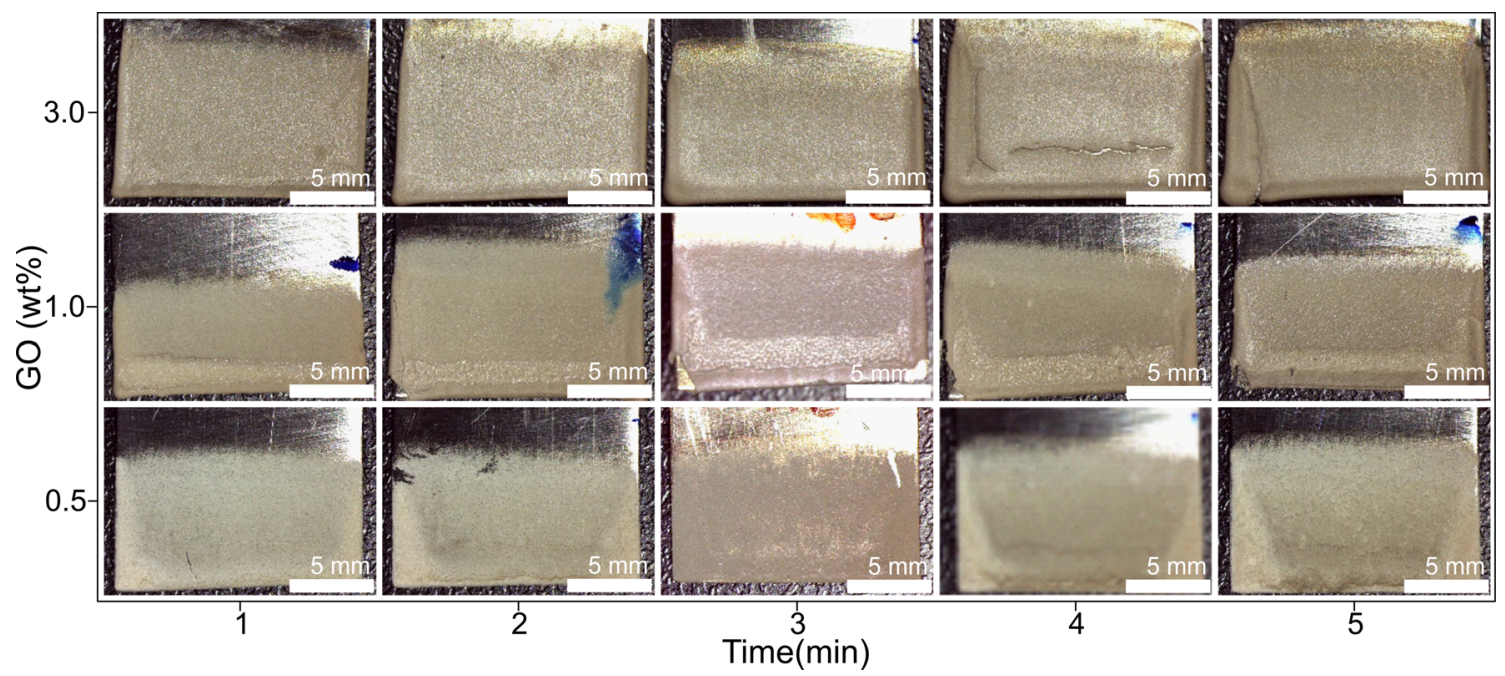

Figure 7 Photographs of coatings prepared from PEEK/GO suspensions with different GO concentration in function of deposition time. The voltage in all cases is $30 \mathrm{~V}$.

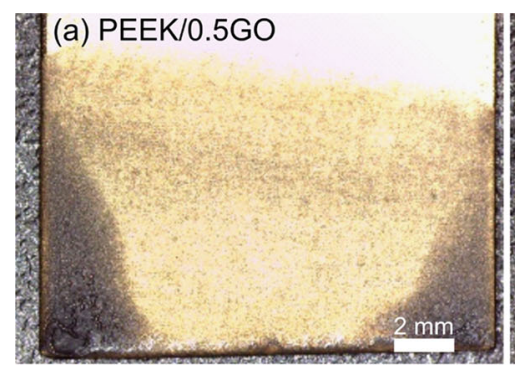

Figure 8 Photographs of PEEK/GO coatings after drying and thermal treatment. The coatings were obtained from suspensions with different GO loadings. The respective GO $\mathrm{wt} \%$ ratio is

morphology with preferential orientation appears on all samples in the polymer-rich regions, which decreases at higher values of GO wt\%; meanwhile, the roughness increases (Fig. $9 \mathrm{~d}-\mathrm{f}$ ). This dendritic morphology was also found in neat PEEK coatings after drying and thermal treatment (PEEK_TT) at the boundaries of the coating (Fig. 10).

Transcrystallinity or surface crystallisation is a condition where nucleation and crystalline growth are affected by an existing surface; as a result, spherulites originate at the surface and grow normal to it. Transcrystallinity has been reported for PEEK on different substrates [76, 80, 93], and more recently on GO [94]. In PEEK/GO composites obtained by injection moulding, a PEEK dendritic morphology similar to that shown in Fig. 10 was observed growing with preferential orientation on a GO flake and it was related to changes in the ratio of the (110) to (200) peak intensities of the bulk XRD patterns [94]. indicated in the sample codification on each image. Electrophoretic parameters: $30 \mathrm{~V}$ and 3-min deposition time.

Analogously, the XRD pattern for the PEEK/ 3.0GO_TT coating (Fig. 11) showed a decrease in the ratio of the (110) to (200) peak intensities in comparison with those of pure PEEK and PEEK_TT, indicating an important increase in the crystalline phase as a result of the thermal treatment in both PEEK_TT and PEEK/GO_TT coatings. The degree of crystallisation for PEEK_TT and PEEK/3.0GO_TT coatings is summarised in Table 5 . Thermal treatment tends to increase the degree of crystallisation of pure PEEK (Tables 3, 5) [77, 81, 83]; nevertheless, graphene appears to have a nucleating effect for the crystallisation of PEEK from the melt and to affect the crystalline growth as well $[94,95]$. In the case of PEEK/ 3.0GO_TT coating, the degree of crystallinity appears to be reduced by the co-continuous GO structure within the PEEK composite (Table 5). A similar effect has been recently reported in a non-isothermal 

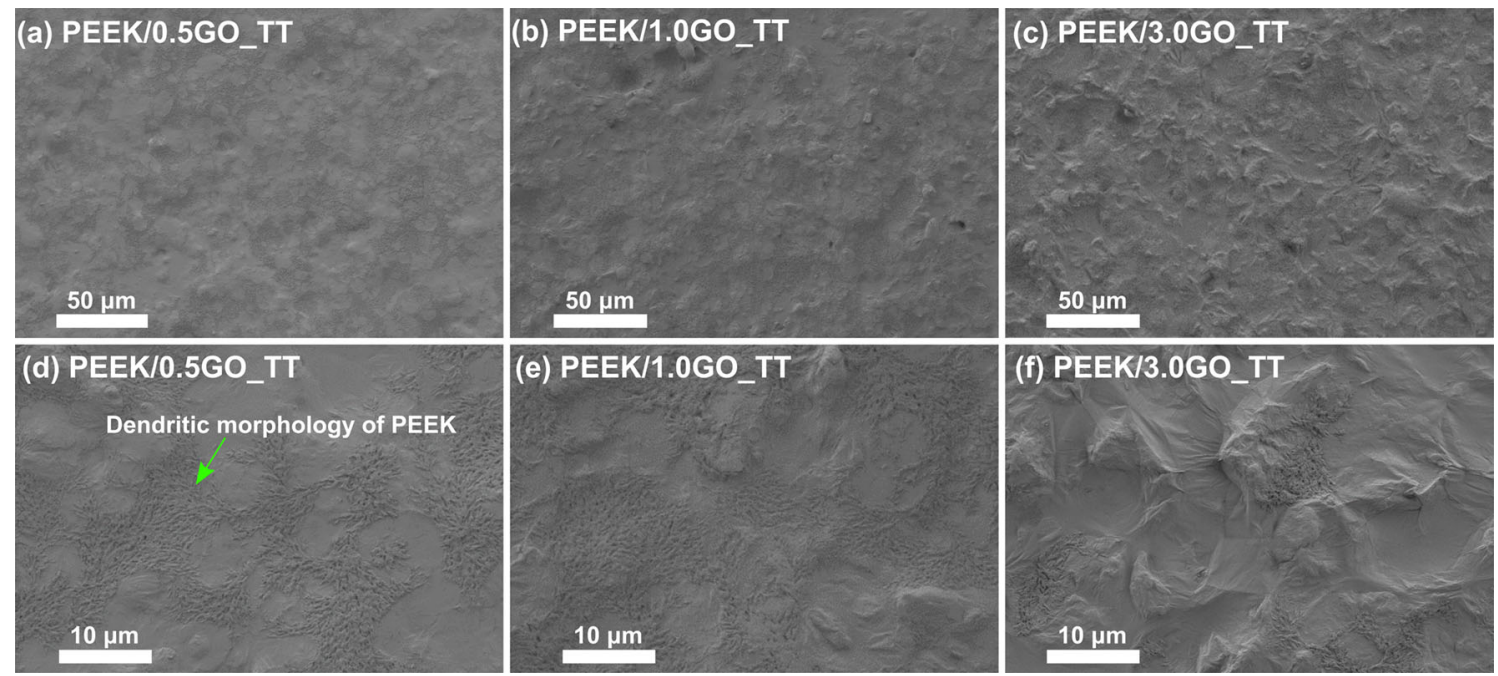

Figure 9 SEM micrographs at different magnifications of PEEK/ GO_TT coatings. These coatings were obtained from suspensions with different GO loadings then subjected to thermal treatment to consolidate the nanocomposite. The respective $\mathrm{GO} \mathrm{wt} \%$ ratio is indicated in the sample codification on each micrograph. Electrophoretic deposition parameters: $30 \mathrm{~V}$ and 3-min deposition time.
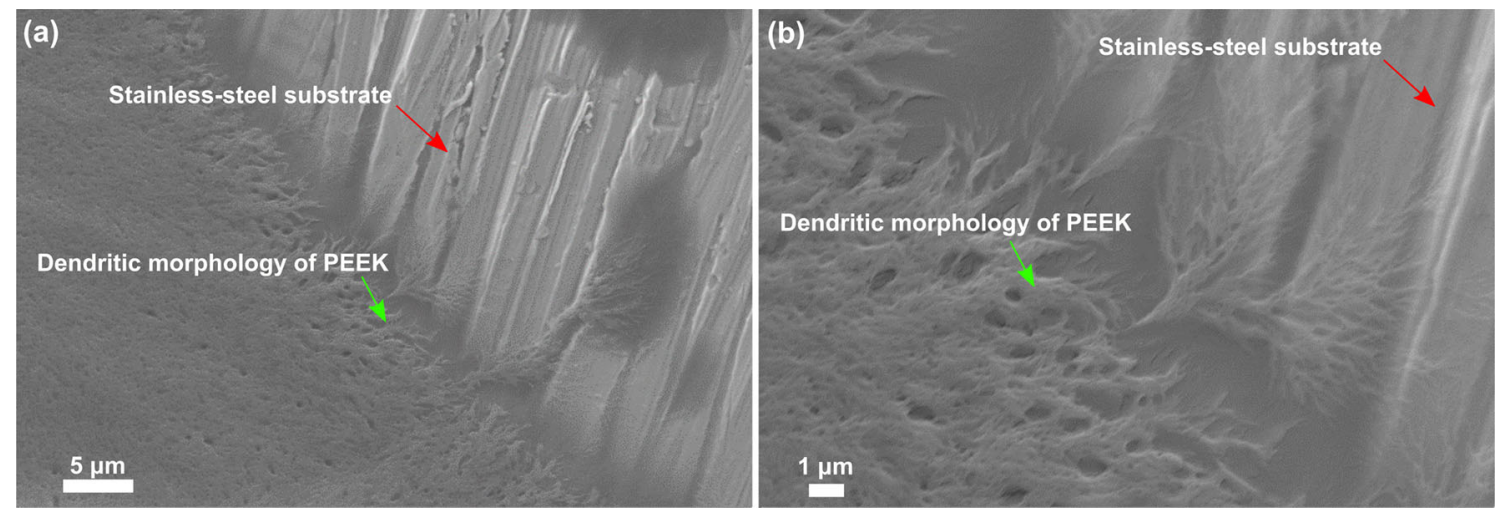

Figure 10 SEM micrographs, at two magnifications, of a neat PEEK coating after drying and thermal treatment (PEEK_TT). A dendritic morphology with preferential orientation is clearly visible at the edges of the coating.

crystallisation study of PEEK/graphene nanoplate composites by synchrotron X-ray diffraction [95].

On the other hand, location and chemical structure of the surrounding polymer, in addition to time and temperature, have been observed to affect the in-situ reduction of GO in polymer matrix composites $[96,97]$. In this respect, the FTIR spectrum of neat GO after thermal treatment, GO_TT, is shown in Fig. 12, and it is compared with those of PEEK_TT and PEEK/3.0GO_TT. In the inset of Fig. 12, the contribution of GO_TT and PEEK_TT to the PEEK/ 3.0GO_TT spectrum is presented. During thermal reduction of $\mathrm{GO}$, the restoration of the aromatic rings gives rise to the band at $1570 \mathrm{~cm}^{-1}$, indicated in the inset of Fig. 12, which is associated with $\mathrm{C}=\mathrm{C}$ stretching vibration of the $\mathrm{sp}^{2}$ hybridised carbon atoms [97]. Additionally, the thermal treatment also induced changes in those bands associated with crystallinity [75-79]. However, there was no sign of the aldehyde band at $1709 \mathrm{~cm}^{-1}$ which is associated with the onset of thermal degradation of PEEK [71], thus confirming an adequate thermal treatment adopted in this work. The degree of crystallisation of PEEK_TT estimated by considering the calibration curve [77] agreed with the XRD measurements (Table 5).

Another important characteristic is that the morphology of the PEEK/3.0GO_EPD coating developed at $10 \mathrm{~V}$ and 1-min deposition time (Fig. 4f) and that of the coating obtained at $30 \mathrm{~V}$ and 3 min deposition 


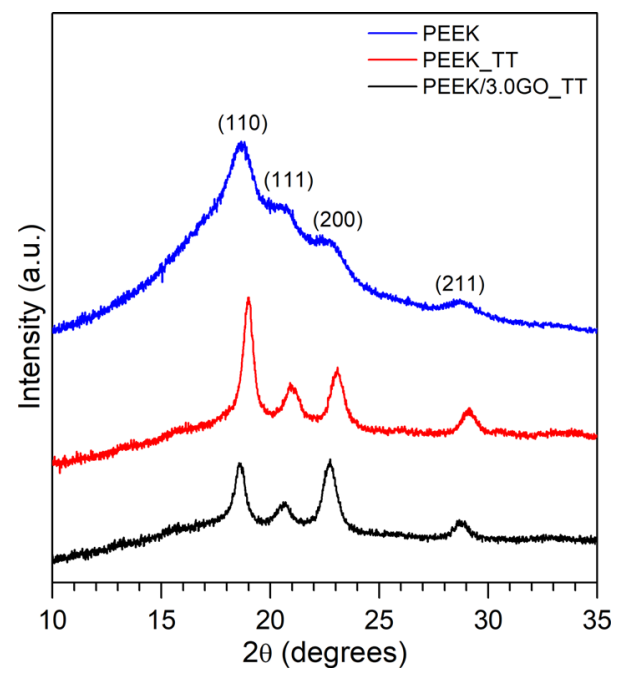

Figure 11 XRD patterns of neat PEEK, PEEK_TT and PEEK/ 3.0GO TT.

time were similar (Fig. 5f). Thereby, it is reasonable to conceive the characteristics of the coating morphology across the cross section as being similar to those on the coating surfaces. This conception of the coating morphology is supported by SEM images of the coating cross sections (Fig. 13), for PEEK/3.0GO_TT prepared at deposition time of $3 \mathrm{~min}$, highlighting the consistent microstructure throughout the EPD film. The texture observed on the upper surface of the films is due to the presence of the wrapped PEEK particles (Fig. 13a, b). In addition, similar to the surface morphology (Fig. 9a-f), both wrapped PEEK particles and PEEK dendrites are observed (Fig. 13c, d).

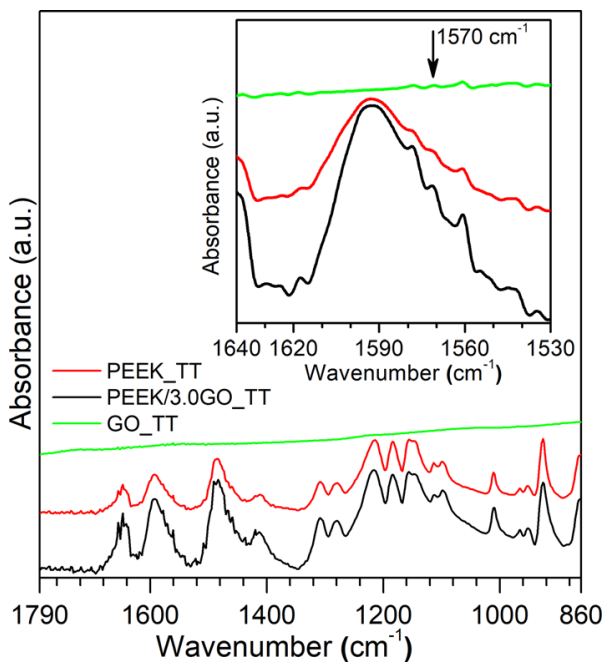

Figure 12 FTIR spectra of GO_TT, PEEK_TT and PEEK/ 3.0GO TT.

\section{Conclusions}

Intense tip sonication of PEEK particle suspensions reduced the particle size and increased the aspect ratio without significantly affecting PEEK chemical structure and degree of crystallinity. The reduced size was considered an advantage for the preparation of PEEK/GO coatings by EPD.

The resulting PEEK suspensions were successfully mixed with GO suspensions and used for the preparation of composite coatings by electrophoretic co-deposition. The resulting microstructure displayed an intimate interaction between the polymer and GO nanosheets homogeneously across and throughout the coatings. The two phases formed a cocontinuous architecture, built around an aligned network of GO, particularly favoured by high GO concentrations. The presence of GO created a texture in the consolidated (heat-treated) polymer films,

Table 5 Estimation of the degree of crystallinity of PEEK and PEEK/3.0GO coatings after thermal treatment according to XRD measurements and calibration curve reported by Cebe et al. [77]

\begin{tabular}{lllll}
\hline Coating & FTIR & & XRD \\
\cline { 2 - 4 } & $\begin{array}{l}\text { Band area ratio } \\
966 \mathrm{~cm}^{-1} / 952 \mathrm{~cm}^{-1}\end{array}$ & $\begin{array}{l}\text { Degree of crystallinity (\%) } \\
\text { from calibration curve [77] }\end{array}$ & $\begin{array}{l}\text { Degree of } \\
\text { crystallinity (\%) }\end{array}$ \\
\hline PEEK_TT & 0.5331 & 37 & 35 \\
PEEK/3.0GO_TT & - & - & 22 \\
\hline
\end{tabular}

The considered MIR bands correspond to $\omega(\mathrm{CH})$ modes 

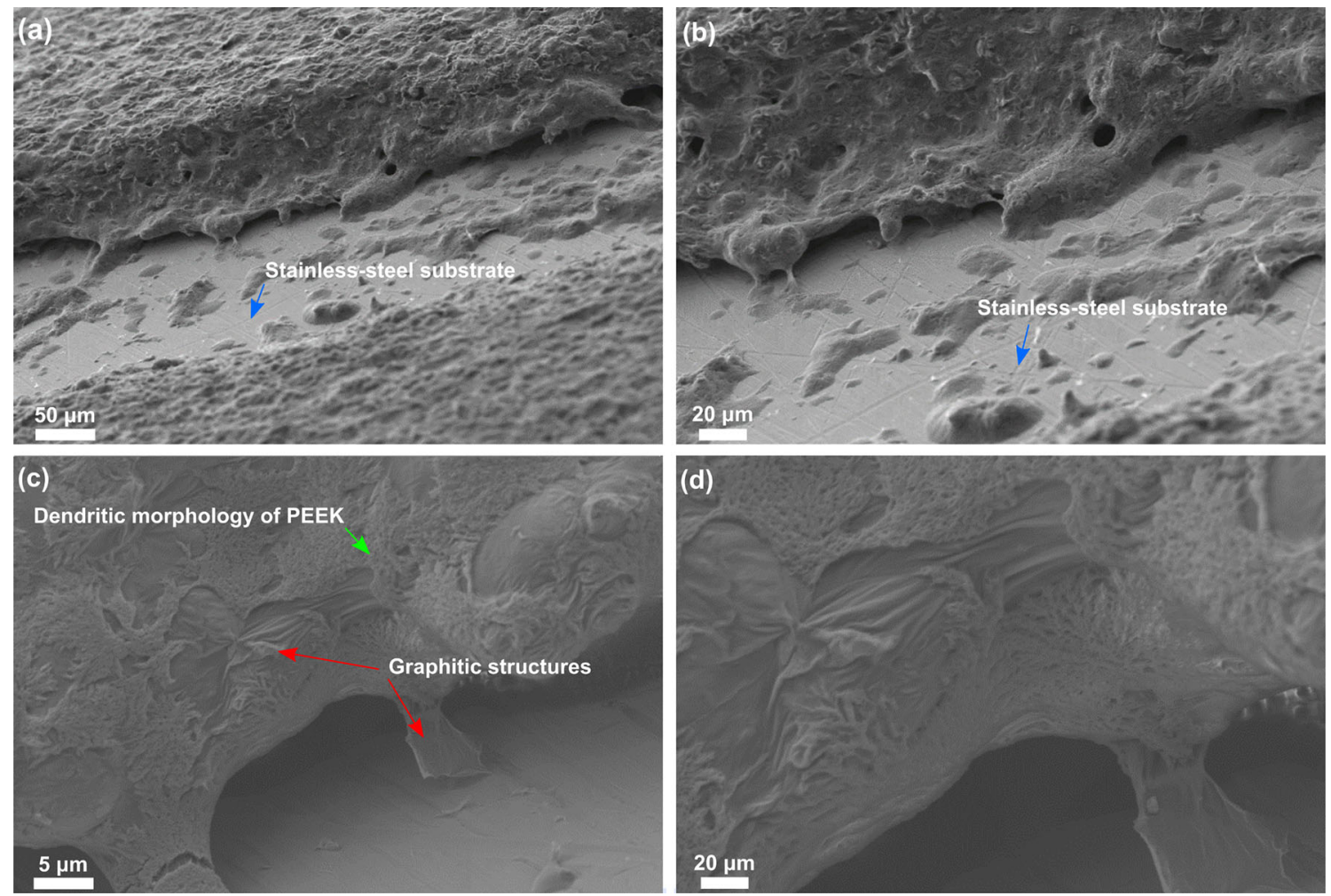

Figure 13 SEM micrographs at different magnifications of the cross section of a PEEK/3.0GO_TT coating. Electrophoretic deposition parameters: $30 \mathrm{~V}$ and 3 min deposition time.

preserving some characteristics of the original PEEK particles, the wrapping effect, as well as nucleating a high degree of PEEK matrix and inducing transcrystallinity. These PEEK/GO nanocomposite coatings obtained by EPD may have promising properties given their unique morphological characteristics. The continuous network of GO nanosheets should provide electrical and thermal conductivity, once the GO is reduced, for example during the heating treatment used to consolidate the polymer. It may also contribute to achieve superior mechanical properties, regarding the elastic modulus, hardness, and other important coating characteristics such as impact and abrasion resistance. The presence of carbon nanofillers in PEEK is known to improve properties both through direct reinforcement and improvement in matrix microstructure [98], particularly crystallinity, as observed here. In this respect, the emphasis of our future work will be the characterisation of the coating physical properties and the relationship of the properties with the micromorphology observed. The deposition of PEEK/GO coatings by EPD is attractive, representing a simple and efficient technology to create useful nanocomposites on a wide range of substrates, including curved surfaces. Such systems may be relevant to applications in industrial and food processing, and in the textile, medical, oil, and gas sectors.

\section{Acknowledgements}

Open Access funding provided by Projekt DEAL. Eduin I. González-Castillo gratefully acknowledges the National Council of Science and Technology (CONACYT, Mexico) for the postdoctoral scholarship given (Reference: 239274) to carry out his project proposal in Germany.

\section{Compliance with ethical standards}

Conflict of interest There is no conflict of interest.

Electronic supplementary material: The online version of this article (https://doi.org/10.1007/s108 53-020-04632-4) contains supplementary material, which is available to authorized users. 
Open Access This article is licensed under a Creative Commons Attribution 4.0 International License, which permits use, sharing, adaptation, distribution and reproduction in any medium or format, as long as you give appropriate credit to the original author(s) and the source, provide a link to the Creative Commons licence, and indicate if changes were made. The images or other third party material in this article are included in the article's Creative Commons licence, unless indicated otherwise in a credit line to the material. If material is not included in the article's Creative Commons licence and your intended use is not permitted by statutory regulation or exceeds the permitted use, you will need to obtain permission directly from the copyright holder. To view a copy of this licence, visit http://creativecommons.org/licen ses/by $/ 4.0 /$.

\section{References}

[1] Kurtz SM (2012) Chapter 1-an overview of PEEK biomaterials. In: Kurtz SM (ed) PEEK biomaterials handbook. William Andrew Publishing, Oxford, pp 1-7

[2] Kurtz SM (2012) Chapter 6-chemical and radiation stability of PEEK. In: Kurtz SM (ed) PEEK biomaterials handbook. William Andrew Publishing, Oxford, pp 75-79

[3] Sobieraj MC, Rimnac CM (2012) Chapter 5-fracture, fatigue, and notch behavior of PEEK. In: Kurtz SM (ed) PEEK biomaterials handbook. William Andrew Publishing, Oxford, pp 61-73

[4] Babrauskas V, Fuoco R, Blum A (2014) Chapter 3-flame retardant additives in polymers: when do the fire safety benefits outweigh the toxicity risks? In: Papaspyrides CD, Kiliaris P (eds) Polymer green flame retardants. Elsevier, Amsterdam, pp 87-118

[5] Wypych G (2012) PEKK polyetherketoneketone. In: Wypych G (ed) Handbook of polymers. Elsevier, Oxford, pp 367-369

[6] Sak A et al (2016) Influence of polyetheretherketone coatings on the $\mathrm{Ti}-13 \mathrm{Nb}-13 \mathrm{Zr}$ titanium alloy's bio-tribological properties and corrosion resistance. Mater Sci Eng C 63:52-61

[7] Boccaccini AR et al (2010) Electrophoretic deposition of biomaterials. J R Soc Interface 7(5):S581-S613

[8] Corni I, Ryan MP, Boccaccini AR (2008) Electrophoretic deposition: From traditional ceramics to nanotechnology. J Eur Ceram Soc 28(7):1353-1367
[9] Sarkar P, Nicholson PS (1996) Electrophoretic deposition (EPD): mechanisms, kinetics, and application to ceramics. J Am Ceram Soc 79(8):1987-2002

[10] Schaefer DW, Justice RS (2007) How nano are nanocomposites? Macromolecules 40(24):8501-8517

[11] Chawla KK (2012) Composite materials Science and engineering, 3rd edn. Springer, New York, p 542

[12] Lubin G (1982) Handbook of composites, 1st edn. Springer, US, New York, p 786

[13] Mai Y-W, Yu Z-Z (eds) (2006) Polymer Nanocomposites. Woodhead Publishing, Sawston, p 608

[14] Dickerson JH, Boccaccini AR (eds) (2012) Electrophoretic deposition of nanomaterials. Nanostructure science and technology. Springer, New York, p 376

[15] Moskalewicz T et al (2019) Effect of the processing and heat treatment route on the microstructure of MoS2/ polyetheretherketone coatings obtained by electrophoretic deposition. J Electrochem Soc 166(6):D151-D161

[16] Diba M et al (2016) Electrophoretic deposition of graphenerelated materials: a review of the fundamentals. Prog Mater Sci 82:83-117

[17] Wu Z-S et al (2009) Field emission of single-layer graphene films prepared by electrophoretic deposition. Adv Mater 21(17):1756-1760

[18] Chen J et al (2016) Reduced graphene oxide deposited carbon fiber reinforced polymer composites for electromagnetic interference shielding. Compos A Appl Sci Manuf $82: 141-150$

[19] Ma Y et al (2018) Electrophoretic deposition of graphenebased materials: a review of materials and their applications. J Materiom 4(2):108-120

[20] Chen Y et al (2010) Electrophoretic deposition of graphene nanosheets on nickel foams for electrochemical capacitors. J Power Sources 195(9):3031-3035

[21] Pan L et al (2019) Enhancement in interfacial adhesion of Ti/ polyetheretherketone by electrophoretic deposition of graphene oxide. Polym Compos 40(S2):E1243-E1251

[22] Wu T et al (2011) Adsorption characteristics of acrylonitrile, p-toluenesulfonic acid, 1-naphthalenesulfonic acid and methyl blue on graphene in aqueous solutions. Chem Eng J 173(1):144-149

[23] Rastogi R et al (2008) Comparative study of carbon nanotube dispersion using surfactants. J Colloid Interface Sci 328(2):421-428

[24] Geim AK, Novoselov KS (2007) The rise of graphene. Nat Mater 6:183-191

[25] Compton OC, Nguyen ST (2010) Graphene oxide, highly reduced graphene oxide, and graphene: versatile building blocks for carbon-based materials. Small 6(6):711-723 
[26] Zhu Y et al (2010) Graphene and graphene oxide: synthesis, properties, and applications. Adv Mater 22(35):3906-3924

[27] Potts JR et al (2011) Graphene-based polymer nanocomposites. Polymer 52(1):5-25

[28] Chee WK et al (2015) Nanocomposites of graphene/polymers: a review. R Soc Chem Adv 5(83):68014-68051

[29] Young RJ et al (2012) The mechanics of graphene nanocomposites: a review. Compos Sci Technol 72(12):1459-1476

[30] Mittal G et al (2015) A review on carbon nanotubes and graphene as fillers in reinforced polymer nanocomposites. J Ind Eng Chem 21:11-25

[31] Du J, Cheng H-M (2012) The fabrication, properties, and uses of graphene/polymer composites. Macromol Chem Phys 213:1060-1077

[32] Hyun DC et al (2013) Ultrasonic breaking of fibers and microparticles into mesoporous particles with high loading of magnetic nanoparticles. Macromol Mater Eng 298(5):575-582

[33] Biamino $\mathrm{S}$ et al (2011) $\mathrm{Al}_{2} \mathrm{O}_{3}-\mathrm{ZrO}_{2}$ nanocomposites produced by solution combustion synthesis followed by ultrasonic milling. J Ceram Process Res 12(2):207-211

[34] Suslick KS et al (1991) Sonochemical synthesis of amorphous iron. Nature 353(6343):414-416

[35] Troia A et al (2009) Sonochemical preparation of high surface area $\mathrm{MgAl}_{2} \mathrm{O}_{4}$ spinel. Ultrason Sonochem 16(1):136-140

[36] Torres-Sanchez C, Corney JR (2008) Effects of ultrasound on polymeric foam porosity. Ultrason Sonochem 15(4):408-415

[37] Martina V, De Riccardis F, Carbone D (2010) A chemometric study of alumina/peek and hydroxyapatite/PEEK suspensions prepared for electrophoretic deposition of multifunctional coatings. Adv Sci Technol 66:29-34

[38] Iveković A et al (2015) Aqueous electrophoretic deposition of bulk polyether ether ketone (PEEK). J Mater Process Technol 223:58-64

[39] De Riccardis MF, Martina V, Carbone D (2013) Study of polymer particles suspensions for electrophoretic deposition. J Phys Chem B 117(6):1592-1599

[40] Ur Rehman MA et al (2018) Electrophoretic deposition of lawsone loaded bioactive glass (BG)/chitosan composite on polyetheretherketone (PEEK)/BG layers as antibacterial and bioactive coating. J Biomed Mater Res Part A 106(12):3111-3122

[41] Baştan FE et al (2018) Electrophoretic co-deposition of PEEK-hydroxyapatite composite coatings for biomedical applications. Colloids Surf B 169:176-182
[42] Clavijo S et al (2014) Characterization of polyetheretherketone particle suspensions for electrophoretic deposition. J Appl Polym Sci 131(20):1-6

[43] Wang C, Ma J, Cheng W (2003) Formation of polyetheretherketone polymer coating by electrophoretic deposition method. Surf Coat Technol 173(2):271-275

[44] Seuss S et al (2012) Electrophoretic deposition of PEEK$\mathrm{TiO}$ composite coatings on stainless steel. Key Eng Mater 507:127-133

[45] Corni I et al (2008) Polyetheretherketone (PEEK) coatings on stainless steel by electrophoretic deposition. Adv Eng Mater 10(6):559-564

[46] Moskalewicz T et al (2018) Electrophoretic deposition, microstructure and selected properties of composite alumina/ polyetheretherketone coatings on the $\mathrm{Ti}-13 \mathrm{Nb}-13 \mathrm{Zr}$ alloy. J Electrochem Soc 165(3):D116-D128

[47] Corni I et al (2009) Electrophoretic deposition of PEEKnano alumina composite coatings on stainless steel. Surf Coat Technol 203(10):1349-1359

[48] Ma J, Wang C, Liang CH (2007) Colloidal and electrophoretic behavior of polymer particulates in suspension. Mater Sci Eng C 27(4):886-889

[49] Boccaccini A et al (2006) Electrophoretic deposition of polyetheretherketone (PEEK) and PEEK/bioglass (R) coatings on NiTi shape memory alloy wires. J Mater Sci 41:8152-8159. https://doi.org/10.1007/s10853-006-0556-Z

[50] Abdulkareem M, Abd Alsalam A, Jabar A (2019) Influence of chitosan on the antibacterial activity of composite coating (PEEK/HAp) fabricated by electrophoretic deposition. Prog Org Coat 130:251-259

[51] Moskalewicz T et al (2017) Electrophoretic deposition, microstructure, and corrosion resistance of porous sol-gel glass/polyetheretherketone coatings on the Ti-13Nb-13Zr alloy. Metall Mater Trans A 48(5):2660-2673

[52] Fiołek A et al (2019) The influence of electrophoretic deposition parameters and heat treatment on the microstructure and tribological properties of nanocomposite $\mathrm{Si}_{3} \mathrm{~N}_{4} / \mathrm{PEEK} 708$ coatings on Titanium alloy. Coatings 9(9):530. https://doi.org/10.3390/coatings9090530

[53] Moskalewicz T et al (2019) Improvement of the Ti-6Al-4V alloy's tribological properties and electrochemical corrosion resistance by nanocomposite TiN/PEEK708 coatings. Metall Mater Trans A 50(12):5914-5924

[54] Ltd, T.M.U. Stainless steel 316L 1.4404 (2018). https://ww w.thyssenkrupp-materials.co.uk/stainless-steel-3161-14404.h tml. Accessed 6 Jan 2020

[55] Taurozzi JS, Hackley VA, Wiesner MR (2011) Ultrasonic dispersion of nanoparticles for environmental, health and safety assessment - issues and recommendations. Nanotoxicology 5(4):711-729 
[56] Miyazawa T et al (2012) Specific heat capacity at constant pressure of ethanol by flow calorimetry. J Chem Eng Data 57(6):1700-1707

[57] Kelley KK (1929) The heat capacities of isopropyl alcohol and acetone from 16 to $298^{\circ} \mathrm{k}$. And the corresponding entropies and free energies. $\mathrm{J}$ Am Chem Soc 51(4):1145-1150

[58] Lightcap IV et al (2012) Electron hopping through single-tofew-layer graphene oxide films. Side-selective photocatalytic deposition of metal nanoparticles. J Phys Chem Lett 3(11):1453-1458

[59] Sheather SJ (2004) Density estimation. Stat Sci 19(4):588-597

[60] Suslick KS, Price GJ (1999) Applications of ultrasound to materials chemistry. Annu Rev Mater Sci 29(1):295-326

[61] Max Basedow A, Heinrich Ebert K (1977) Ultrasonic degradation of polymers in solution. Phys Chem 22:83-148

[62] Retamal Marin RA-O et al (2018) Effects of sample preparation on particle size distributions of different types of silica in suspensions. Nanomaterials 8:454. https://doi.org/10.339 0/nano8070454

[63] Baig $Z$ et al (2018) Investigation of tip sonication effects on structural quality of graphene nanoplatelets (GNPs) for superior solvent dispersion. Ultrason Sonochem 45:133-149

[64] Hilding $\mathbf{J}$ et al (2003) Dispersion of carbon nanotubes in liquids. J Dispers Sci Technol 24(1):1-41

[65] Price GJ, White AJ, Clifton AA (1995) The effect of highintensity ultrasound on solid polymers. Polymer 36(26):4919-4925

[66] Tang ESK, Huang M, Lim LY (2003) Ultrasonication of chitosan and chitosan nanoparticles. Int $\mathrm{J}$ Pharm 265(1):103-114

[67] Paulusse JMJ, Sijbesma RP (2006) Ultrasound in polymer chemistry: revival of an established technique. J Polym Sci Part A Polym Chem 44(19):5445-5453

[68] Hay JN, Kemmish DJ (1987) Thermal decomposition of poly(aryl ether ketones). Polymer 28(12):2047-2051

[69] Jonas A, Legras R (1991) Thermal stability and crystallization of poly(aryl ether ether ketone). Polymer 32(15):2691-2706

[70] Patel P et al (2010) Mechanism of thermal decomposition of poly(ether ether ketone) (PEEK) from a review of decomposition studies. Polym Degrad Stab 95(5):709-718

[71] Naffakh M et al (1999) Thermal decomposition of technological polymer blends.1. Poly(aryl ether ether ketone) with a thermotropic liquid crystalline polymer. Polym Degrad Stab 66:405-413

[72] Koopal LK, Lyklema J (1975) Characterization of polymers in the adsorbed state by double layer measurements. The silver iodide + poly(vinyl alcohol) system. Faraday Discuss Chem Soc 59:230-241

[73] Liu J et al (1997) Aggregation and dispersion of colloidal suspensions by inorganic surfactants: effect of chemical speciation and molecular conformation. J Phys Chem B 101(41):8264-8269

[74] Silberberg A (1962) The adsorption of flexible macromolecules. Part I. The isolated macromolecule at a plane interface. J Phys Chem 66(10):1872-1883

[75] Chalmers JM, Gaskin WF, Mackenzie MW (1984) Crystallinity in poly(aryl-ether-ketone) plaques studied by multiple internal reflection spectroscopy. Polym Bull $11: 433-435$

[76] Nguyen HX, Ishida H (1986) Molecular analysis of the melting behaviour of poly(aryl-ether-ether-ketone). Polymer 27(9):1400-1405

[77] Cebe P, Chung SY, Hong S-D (1987) Effect of thermal history on mechanical properties of polyetheretherketone below the glass transition temperature. J Appl Polym Sci 33(2):487-503

[78] Damman P et al (1994) Crystallinity of poly(aryl ether ether ketone) by vibrational spectroscopy. Macromolecules 27:1582-1587

[79] Chalmers JM et al (1998) Fourier transform infrared microscopy: some advances in techniques for characterisation and structure-property elucidations of industrial material. The Analyst 123:579-586

[80] Waddon AJ, Hill MJ, Keller A (1987) On the crystal texture of linear polyaryls (PEEK, PEK and PPS). J Mater Sci 22:1773-1778. https://doi.org/10.1007/BF01132406

[81] Blundell DJ, Osborn BN (1983) The morphology of poly (aryl-ether-ether-ketone). Polymer 24(8):953-958

[82] Hay JN, Langford JI, Lloyd JR (1989) Variation in unit cell parameters of aromatic polymers with crystallization temperature. Polymer 30(3):489-493

[83] Reitman M et al (2012) Chapter 4-morphology and crystalline architecture of polyaryletherketones. In: Kurtz SM (ed) PEEK biomaterials handbook. William Andrew Publishing, Oxford, pp 49-60

[84] Hardle W, Marron JS, Wand MP (1990) Bandwidth choice for density derivatives. J R Stat Soc Ser B (Methodological) 52(1):223-232

[85] Bai L et al (2018) Kinetic control of graphene localization in co-continuous polymer blends via melt compounding. Langmuir 34(3):1073-1083

[86] Gaska K et al (2017) Gas barrier, thermal, mechanical and rheological properties of highly aligned graphene-LDPE nanocomposites. Polymers 9:294. https://doi.org/10.3390/ polym 9070294 
[87] Hiura H, Miyazaki H, Tsukagoshi K (2010) Determination of the number of graphene layers: discrete distribution of the secondary electron intensity stemming from individual graphene layers. Appl Phys Express. https://doi.org/10.1143/A PEX.3.095101

[88] Zarbov M et al (2006) EPD of metallic silver particles: problems and solutions. Key Eng Mater 314:95-100

[89] Mori Y et al (2017) Thin film structure of titania nanoparticles prepared by electrophoretic deposition. Chem Eng Trans 57:1507-1512

[90] Zitzenbacher G et al (2018) Calculation of the contact angle of polymer melts on tool surfaces from viscosity parameters. Polymers 10(1):38. https://doi.org/10.3390/polym10010038

[91] Vallés C et al (2014) The rheological behaviour of concentrated dispersions of graphene oxide. J Mater Sci 49(18):6311-6320. https://doi.org/10.1007/s10853-014-835 6-3

[92] Papageorgiou DG et al (2019) Hybrid poly(ether ether ketone) composites reinforced with a combination of carbon fibres and graphene nanoplatelets. Compos Sci Technol 175:60-68
[93] Regis $M$ et al (2017) Characterization of thermally annealed PEEK and CFR-PEEK composites: Structure-properties relationships. Polym Degrad Stab 136:121-130

[94] Tewatia A et al (2017) Characterization of melt-blended grapheme-poly(ether ether ketone) nanocomposite. Mater Sci Eng B 216:41-49

[95] Alvaredo Á et al (2019) Non-isothermal crystallization behavior of PEEK/graphene nanoplatelets composites from melt and glass states. Polymers (Basel) 11(1):124

[96] Glover AJ et al (2011) In situ reduction of graphene oxide in polymers. Macromolecules 44(24):9821-9829

[97] Ren Y et al (2018) Online tracking of the thermal reduction of graphene oxide by two-dimensional correlation infrared spectroscopy. Vib Spectrosc 96:32-45

[98] Sandler J et al (2002) Carbon-nanofibre-reinforced poly(ether ether ketone) composites. Compos A Appl Sci Manuf 33(8):1033-1039

Publisher's Note Springer Nature remains neutral with regard to jurisdictional claims in published maps and institutional affiliations. 